\title{
LA LECTURA DEL TIMEO EN CHARTRES: TEODORICO DE CHARTRES Y GUILLERMO DE CONCHES
}

\author{
Francisco Tauste Alcocer \\ Profesor de Filosofía. I.B. Barcelona
}

El humanismo chartreano se manifiesta especialmente en una preocupación por las Artes Liberales, que son consideradas no sólo como la base cultural indispensable para los estudios filosóficos sino también como propedéutica para el estudio de la Revelación, haciendo realidad ese ideal tan sentido por los Padres de la Iglesia, de intentar una armonización entre la cultura pagana y el pensamiento cristiano.

Pero antes es preciso alcanzar una armonía previa: la cohaerentia artium, de modo que la eloquentia no se entiende como un ejercicio retórico vacío sino como instrumento al servicio de la Sapientia . En esto consiste la cultura humanista del momento, en mantener un equilibrio entre el pensamiento y la palabra, entre la ratio y el verbum. Un saber que recupera un sentido cívico, hasta ahora olvidado, ya que la cultura tenía un carácter más monástico y rural que urbano.

Es en este contexto cultural en el que en la primera mitad del siglo XII florecen una serie de comentarios y glosas a autores como Boecio, Macrobio, Marciano Capella y Platón cuyo Timeo en la traducción parcial de Calcidio es objeto de toda clase de interpretaciones.

Con el estudio de estos auctores intentan una aproximación y, a ser posible, un acuerdo doctrinal entre los pensadores paganos, en este caso Platón, y la Escritura. Y en esta empresa cuentan con ilustres predecesores como San Agustín y Boecio.

La lectura del Timeo les lleva inmediatamente al Génesis, con el fin de abordar la doctrina de la creación secundum physicam. Tal es el cometido de Teodorico de Chartres. Por su parte, Gillermo de Conches, al glosar el Timeo, tiene presente el contenido de la Revelación. En definitiva, podemos afirmar que la lectura del Timeo en Chartres es siempre una lectura en paralelo, teniendo muy cerca la narración del origen del mundo del primer capítulo del Génesis.

Tal lectura en paralelo quedaría incompleta si olvidáramos un referente importantísimo a la hora de hablar de Chartres: la ciencia greco-árabe, junto con el descubrimiento de un aristotelismo naturalista cuyo efecto antiespeculativo no se hace esperar, hasta el punto de que 
autores como Conches o Abelardo tengan que enfrentarse con corrientes más idealistas como los Victorinos cuando no con las condenas eclesiásticas.

EI naturalismo chartreano que resulta de estas influencias se inclina más por una interpretación optimista del mundo, más en consonancia con la tradición del Timeo cuyo «dualismo» siempre se consideró de forma atenuada, debido al contacto con las doctrinas medioplatónicas y estoicas.

Su visión libre de la Revelación bíblica les lleva a ver en el relato del Génesis una confirmación de su optimismo. En efecto, al término de cada jornada de la creación dice el hagiógrafo: «y vio Dios que era bueno», frase que se repite hasta siete veces a lo largo de este primer capítulo. No cabe duda de que esta insistencia en la bondad de la creación les llevó a conectar más fácilmente no sólo con la tradición optimista del Timeo sino también con las aportaciones científicas del momento que contextualizaban la lectura de ambas obras dentro de la línea claramente comprometida con el avance del saber y del conocimiento del mundo. La repercusión doctrinal de semejante actitud naturalista se ha de manifestar en una valoración más positiva de las realidades mundanas, así como en un énfasis especial en su autonomía relativa en relación con el Creador.

Lo que más les llamó la atención a los antiguos para sentir esa admiración por el Universo fue ese orden inteligente cuyos efectos armónicos remitían a una causa sabia autora de semejante belleza mundana. Aunque en el Timeo, el autor de este orden, el Demiurgo, no figura en el máximo rango de la escala del ser, sin embargo su imagen fue asociada a la del Creador bíblico ya que Palatón se planteó desde el principio de su diálogo la tarea de encontrar al «Productor y Padre de todo» (Tim . 28 c). Y así, los Padres trataron de interpretar en sentido creacionista pasajes del Timeo que podían ser interpretados de forma diferente. Más aún, se atrevieron a afirmar que ya Platón llegó a atisbar algunos elementos de la Revelación de forma todavía confusa. Tal es el parecer de Clemente de Alejandría en sus Stromata cuando afirma: «¿Qué otra cosa es Platón sino Moisés que se expresa en ático?».

Toda esta empresa la llevaron a cabo los chartreanos, movidos no por una preocupación apologética especial sino porque estaban convencidos de que se podía lograr una concordia doctrinal entre ambas tradiciones, sobre todo en un asunto tan importante y tan vinculado al desarrollo científico como sigue siendo aún hoy el relativo a la génesis del cosmos.

En definitiva, la lectura del Timeo proporcionó a los pensadores de Chartres y a los poetas de finales del XII una forma alegórica de entender la estructura del mundo físico, cuyos contenidos filosóficos estaban envueltos con el velo del mito. Y así el Timeo podía ser entendido como un gran integumentum o como una serie casi continua de integumenta. Esta visión poética del Timeo que comprendía a éste como un «Mythos» no se hacía en virtud de una opción irracionalista sino con el convencimiento plena de que sólo una lectura alegórica podía evitar al filósofo o al teólogo el cometer una serie de errores doctrinales al tratar de acomodar la filosofía platónica a la verdad revelada.

Este método de interpretación del Timeo no era nada nuevo sino que contó con un precedente en las escuelas medioplatónicas y neoplatónicas. En realidad se remonta a 
Aristóteles que en De Caelo (I, 10, 179b 32-280a 10) llegó a afirmar que sostener que el mundo ha sido engendrado no implica aceptar que ha nacido en el tiempo, sino que tal afirmación se utiliza como un procedimiento didáctico con el fin de comprender mejor la constitución del mundo.

Como vemos, los antiguos se aproximaron a la idea de creación por una finalidad didáctica. Este fue el parecer de Jenócrates, Teofrastro, Plutarco, Atico, Plotino, Porfirio, Jámblico y Proclo. Incluso Calcidio participa también de esta opinión.

«... quorum animis sic institutis difficile persuadeatur mundi esse auctorem Deum, nisi eum tamquam opifex aliquis manibus ceterorumque artium molitione construxerit» (In Timeum cap. 26)

$Y$ en otro texto insiste en esta idea de la génesis del mundo: «origo eius causativa, non temporaria», afirmando al propio tiempo su eternidad: «sic mundus sensilis, licet et corporeus, a Deo tamen factus atque institutus, aeternus est» (Op. cit. cap. 23. Debido a esta lectura alegórica, Calcidio entendía perfectamente que la narración sobre el origen del mundo no implicaba un comienzo absoluto en el tiempo. Y Guillermo de Conches, por influjo de Boecio, afirma algo parecido: «Creator non praecedit creaturam temporis quantitate, sed simplicitate naturae» (Glosas a Boecio, ed. Parent. 134).

La aproximación doctrinal entre el pensamiento pagano y el cristianismo no implica un acercamiento respecto a los contenidos teóricos sino más bien su propia difuminación a través del lenguaje alegórico. La ficción literaria contribuye así a este concordismo no exento de inexactitudes verbales, interpretaciones erróneas y afirmaciones cercanas a la herejía. Pero es que la realidad de ambas tradiciones no ofrece otras posibilidades de acuerdo.

\section{TEODORICO DE CHARTRES: De sex dierum operibus}

Uno de los grandes representantes del humanismo chartreano fue Teodorico de Chartres que enseñó en esta escuela primero como profesor, después como canciller desde 1120 hasta 1153. Los elogios que recibe por parte de sus contemporáneos son una muestra más de su preocupación por las Artes Liberales y su dedicación a la filosofía platónica. Hermann de Carintia le dedica su traducción del Planisferio de Ptolomeo (1143) y Bernardo Silvestre su Cosmografia, escrita entre 1145 y 1153.

El objetico que se plantea Teodorico en su Tractatus es realizar una lectura secundum physicam et ad litteram del primer capítulo del Génesis con el fin de justificar los contenidos del texto sagrado mediante una investigación racional de los datos físicos que aparecen en dicho texto. Por eso, lejos de constituir una lectura alegórica, lo que busca es el sensus litterae historialis, es decir, el sentido literal del texto con el que poder reflejar más fielmente lo que aconteció en los comienzos del mundo.

Se trata de hacer una lectura diferente del Timeo, más bien inversa. Aquí el texto platónico se utiliza para ilustrar la perspectiva naturalista de la interpretación del texto sagrado. Pero no 
hay que olvidar el ámbito en el que estamos situados: el de la exágesis bíblica. Por ello son justas las preguntas que podemos formularnos de acuerdo con las afirmaciones de algunos intérpretes como E. Maccagnolo o P. Duhem: ¿Es el Tractatus una teología racional (Maccagnolo) o una obra física (Duhem)?

Desde luego que cuando utilizamos el término 'física' lo hacemos en sentido boeciano, es decir, se trata de un conocimiento que se alcanza con el uso de la razón con tal de que conecte luego con la teología. Estamos ante una obra que ofrece una vertiente teológica pero como conclusión o desenlace final, después de haber pasado por la física y la matemática, conforme a la división de las ciencias tan utilizada por el aristotelismo.

No es, pues, la teología natural propia del Medievo lo que constituye el discurso filosófico de Teodorico sino una teología mucho más antigua que arranca de los pitagóricos y atraviesa toda la tradición platónica hasta llegar a la Antigüedad tardía. Nadie como él en este momento llegó a captar la relación estrecha entre Pitágoras y Platón, relación que utiliza para justificar determinadas interpretaciones del platonismo como la negación de la eternidad de la materia.

No estamos, pues, ante una apologética esquemática sino ante un pensamiento sistemático, fascinado por la incesante capacidad generativa de la naturaleza cuya razón última sólo puede encontrarse en el Creador.

Las partes de este Tractatus son tres: un estudio sobre las causas y el orden de las secuencias temporales en la génesis del mundo, un análisis detallado de la exposición del Génesis y una consideración final sobre Dios. En el prólogo nos dice que deja de lado el sentido alegórico y moral del texto ya que su propósito es la cognitio Dei ex facturis suis, que fue lo que pretendió Moisés al escribir este capítulo.

En la primera parte establece ya desde el comienzo las funciones propias de cada una de las personas de la Trinidad en la creación, que se corresponden con la causa eficiente, formal y final. La causa eficiente es Dios Padre, el que en el principio creó caelum et terram. La causa formal se identifica con la Sabiduría de Dios o el Logos joánico ( la segunda persona de la Trinidad) que contiene desde toda la eternidad las formas de las cosas que se crearán posteriormente. Por último, la causa final está representada por la Bondad del Credor o Espíritu Santo, ejemplificada por la formula insistente: «et vidit Deus quod esset bonum». La causa material está representa por los cuatro elementos.

Pero quizás el tema más importante de esta primera parte es el relativo al orden del tiempo: lo obra de estos seis días es el paso de un orden imperfecto (el propia de la materia primordialis o caos primitivo) a un orden más perfecto (ornatus ), más que la sustitución del desorden por el orden. Ahora bien, en el Eclesiástico 18,1 se nos dice: «Qui vivit in aeternum creavir omnia simul», mientras que en el Exodo 20,11 se habla de que en seis días «operatus est Dominus». ¿Cómo compaginar estos textos? Teodorico trata de clarificar esta aparente contraposición, al afirmar que la primera cita se refiere a la creación de la materia primordial, mientras que la segunda señala el tiempo en el que transcurre la distinctio formarum. Quedan confirmados en estas citas los dos grandes momentos de la creación: la génesis de la materia y el ornatus.

El primer versículo: «In principio creavit Deus caelum et terram» se refiere a la creación de la materia originaria, junto con los cuatro elementos. Los representados por el término 
«caelum» son el fuego y el aire, que tienen la propiedad de ser ligeros, mientras que la «terra» representa al agua y a la tierra que son pesados. Ahora bien, la pesadez («tarditas») de estos elementos se debe al movimiento rápido y a la agitación perpetua de los elementos ligeros que los rodean. La movilidad característica de los elementos ligeros postula la existencia de un punto de apoyo sólido que no puede estar representado más que por los elementos pesados y ligeros en virtud de la cual unos no pueden existir sin los otros. Esta teoría da la razón a Moisés, para quien los cuatro elementos existieron desde siempre.

El segundo versículo: «Terra autem inanis et vacua et tenebrae erant super faciem abyssi» se presenta a discusión. ¿Qué quiere decir que la tierra estaba confusa y vacía? ¿Se trata de la confusión de la materia primordial? Pero, ¿por qué este desorden aparente ha de preceder a la manifestación de la ornatio? Para que se manifieste con claridad la Bondad y la Sabiduría del Creador, responde Teodorico. Sin embargo esta opinión no es compartida por Guillermo de Conches cuyo razonamiento resulta más consistente y mucho más realista:

«Inconveniens est ratio quam inducunt, scilicet Deum ad hoc fecisse ut ostenderet quanta rerum confusio foret nisi bonitas eius ordinaret. Cui ostenderet? Angelo? Sed angelus ex natura et gratia divinam voluntatem innotescit. Homini? Sed nondum erat homo ut homini ostenderet facta eorum, nisi usque ad hominem servarentur. Sed ante hominis creationem ordinata sunt» (Phil. mundi PL 172, col 53d).

Esta postura más matizada de Conches nos puede ofrecer una pista para entender la ordenación de la materia primordial. En medio de la confusión aparente de los elementos, se oculta un orden real puesto por Dios en la materia para que a través de su maduración en el tiempo (los seis días) y de su desarrollo de acuerdo con las leyes de la naturaleza origine las diferentes formas de seres que existen en el universo.

Analicemos sucintamente la obra de estos seis días en el Tractatus de Teodorico. Los elementos ligeros situados en el cielo no quedaron inmóviles sino que comenzaron a moverse circularmente de forma que la primera revolución circular constituyó el día primero, en el curso del cual el fuego comunica su primera propiedad -la luminosidad-al aire y surgió la luz. Pero el fuego además posee una segunda propiedad: el calor que sólo se puede comunicar o al agua o a la tierra; en este caso se la comunicó al agua creando el firmamento en el segundo día. Veamos cómo se formó.

Como consecuencia de esta acción del calor ígneo, el elemento líquido que estaba entre la tierra y la luna se encontró distribuido en dos partes:

- una quedó en la superficie de la tierra (aqua labilis)

- otra fue elevada por encima del aire (aqua vaporaliter suspensa)

El aire fue situado, pues, como elemento divisorio de las aguas superiores e inferiores, es decir, como firmamento.

En el tercer día el calor hizo disminuir las aguas inferiores y emergieron los continentes. Este calor transmitido a la tierra junto con la humedad que ya poseía hizo a ésta capaz de producir hierbas y árboles frutales. 
En el cuarto día aparecen los astros en el firmamento como condensación de las aguas superiores. Desde ahora la acción del fuego celeste se unirá a la acción de los astros en la formación de los restantes seres.

En la quinta jornada las estrellas, debido a su movimiento incesante, hicieron que el calor inicial tuviera un carácter vital, lo cual produjo los animales marinos y las aves, y en la tierra, reptiles, ganados y bestias terrestres. $\mathrm{Y}$ en medio de estos animales terrestres es hecho el hombre a imagen y semejanza de Dios en el sexto día.

Aunque en cada jornada aparece una referencia a la actividad creadora, está también presente una explicación racional autónoma en el juego de las causas segundas que entran en funcionamiento a partir de la creación de la materia primordialis .

Después de la sexta jornada el Creador descansó. Los monstruos que aparecieron posteriormente se debieron a las causas seminales que Dios puso en los seres creados, causas que han producido los seres que han venido a la existencia posteriormente.

La última parte del Tractatus es un resumen de sus tesis de teología racional basada en sus probationes arithmeticae de origen boeciano. La temática de esta parte es una muestra de que lo que pretende Teodorico con su cosmogonía es realizar un itinerarium in Deum partiendo de sus obras. En definitiva, un accesus a la teología tan común a tantos pensadores medievales desde la Patrística. Lo que llama la atención en su explicación de la Obra de los seis días son sus continuas referencias a esta tradición optimista del Timeo a través del tratamiento de una serie de temas como el del ignis artifex, las seminales causae, el papel de los astros en la generación de los seres del mundo sublunar, la materia primordialis y el Alma del mundo.

La armonía del mundo aparece en este discurso teológico-racional como huella de un Dios inescrutable que sólo se da a conocer ad extra a través de este orden mundano cuya contemplación nos produce un sentimiento religioso que anhela acceder a ese Uno que está más allá de todo ser. Pero semejante atracción hacia lo alto no representa nunguna forma de escape de nuestra situación presente en el mundo sino que más bien supone una celebración mundana de la obra de Dios con todas sus implicaciones morales y sociales. No olvidemos que Conches define al Timeo como un tratado sobre la naturalis iustitia .

$\mathrm{La}$ adjudicación a cada una de las personas trinitarias de una serie de funciones cósmicas analizando el papel de la Trinidad en la Creación, no evita del todo una cierta asimilación mecánica de las personas a la tríada platónica, con lo que supone de cuestionamiento de la unidad de Dios.

Resumiendo, la cosmogonía de Teodorico, aunque mediatizada por afanes teológicos, es una muestra más de esa celebración de la Naturaleza como fuerza generadora de los seres, tan extendida entre los autores de Chartres.

\section{GILLERMO DE CONCHES: Glosae in Timeum}

Sobre la vida de Guillermo de Conches poco podemos decir. Se sabe que nació en Conches, cerca de Normandía, a finales del siglo XI, y que recibió su formación filosófica en 
Chartres bajo el magisterio de Bernardo de Chartres que lo familiarizó con el método de lectura de los auctores clásicos: la lectio philosophorum, llegando a ser maestro en esta escuela y formando sus propios discípulos, como Juan de Salisbury, desde 1120 hasta más allá de la mitad de siglo. Escribió dos tipos de obras: los tratados sistemáticos y las glosas. Aunque esta división obedece a la estructura formal de su producción no debemos entenderla como si hubiera que establecer una separación entre unas y otras, ya que las relaciones mutuas son constantes.

Las glosas de Guillermo tienen un interés historiográfico muy claro, ya que a través de ellas podemos saber cómo era la enseñanza impartida en Chartres en la primera mitad del siglo XII bajo la dirección del maestro Bernardo. Esta consistía en poner a los alumnos en contacto con los textos clásicos a través de la lectio. En este sentido, las glosas constituyen la plasmación literaria de esta experiencia educativa.

Sus Glosae in Timeum constituyen una obra de madurez, una vez que ha escrito sus obras sistemáticas. Nadie duda de su importancia debido al papel que desempeñó el Timeo en la cosmovisión chartriana. Efectivamente Gillermo piensa que Platón ha tratado en este diálogo todas las ramas del saber filosófico: la filosofía moral y la filosofía teorética.

Con respecto al método de las glosas hay que insistir en que refleja la propia lectura practicada en la escuela. Se sigue el texto literal del diálogo platónico en la versión fragmentaria del Calcidio, sin perder de vista el conjunto de la obra. La mecánica del comentario es bastante sencilla: anuncia en primer lugar el fragmento que trata de comentar y expone a continuación de forma sistemática el contenido doctrinal del pasaje para finalizar con una explicación del texto frase por frase.

Analizaremos aquellos temas más sobresalientes que guardan una relación especial con esta lectura en paralelo que hemos visto en Teodorico.

El tratado sobre las causas nos presenta las funciones específicas de cada persona trinitaria dentro de la tarea creadora. La causa eficiente está representada por la esencia divina, la formal por la Sabiduría y la final por la Bondad, mientras que la causa material está representada por los cuatro elementos que se encuentran confuse en la materia primordial. En líneas generales se mantiene el esquema de Teodorico.

Se trata de una divisio bimembris de las causas de acuerdo con la división de los seres establecida en Timeo 27d: en un miembro están las causas que han existido siempre y que carecen de generación: causas eficiente, formal y final, mientras que en el otro se encuentran aquellas realidades sometidas a la generación como son la causa material y los efectos de estas cuatro causas.

Así pues, la esencia divina como causa eficiente del mundo es omnium creatrix, la causa formal es aquella según la cual el Creador formó al mundo, de acuerdo con la metáfora platónica del artesano:

«Ut enim faber volens aliquid fabricare, prius in mente disponit, postea, quaesita materia, iuxta mentem suam operatur, sic Creator antequam aliquid crearet, in mente illud habuit, deinde opere illud adimplevit» (Glosae. Edic. Jeauneau XXXII, p. 98). 
$\mathrm{Al}$ abordar la creación, utiliza la definición de mundo de Apuleyo: «ornata ordinatio, dei munere, deorum recte custodia» (De mundo, 7-8). Si el mundo es una ordinata collectio creaturarum, quiere decir que ha sido engendrado de acuerdo con Timeo 28a: "gignitur ex aliqua causa necessario». Estas causas no pueden ser otras que las cuatro sustanciales que vimos antes más dos accidentales: el tiempo y el espacio.

Pero no toda obra generadora es fruto de la acción del Creador, ya que, siguiendo a Calcidio toda obra, «vel est opus Creatoris, vel opus naturae, vel artificis imitantis naturam» (In Timeum cap. 23). La obra del Creador ha consistido fundamentalmente en la creación de la materia primordial junto con los cuatro elementos.

Pero al lado de la acción del Creador, un pensador de Chartres no puede olvidar la acción generadora de la Natura que es definida como una «vis rebus insita similia de similibus operans» (Glosae, XXXVII p. 104). Por otro lado, la obra técnica propia del artesano se realiza debido a una carencia o necesidad del individuo y consiste en la fabricación de vestidos contra el frío, en la construcción de viviendas etc. De acuerdo con Aristóteles, la obra técnica naturam imitatur.

Este mundo es imagen o simulacrum de la Sabiduría divina, y en cuanto está hecho por Dios es honestum.

El mundo es representado per integumentum por el dios Pan, cuyo nombre significa etimológicamente «todo». Efectivamente el mundo que nos describe la fábula de Pan anhela la concordia, porque en ella reside su vida, la continuidad de su existencia, ya que «si fieret discordia elementorum, dissolveretur et mundus» (Op. cit. XXXIX, p. 107).

En Timeo 28b-c se plantea si el mundo ha tenido un comienzo o ha sido siempre así. Si el mundo ha sido engendrado por alguna causa, ha tenido un principio porque ha sido hecho «ex preiacente materia ab aliquo", pero resulta difícil encontrar a su autor, al menos en esta vida, ya que no existe una manifestación directa de su propia realidad. Por ello, hemos de imaginarnos sus acciones, partiendo de las craturas.

La frase de Timeo 29a: «Certe dubium non est, ad cuius modi exemplum animadverterit mundani operis fundamenta constituens, utrum ad immutabile perpetuamque obtinens proprietatem an ad factum et elaboratum», le da pie para tratar la distintición boeciana entre eterno y perpetuo, términos a los que añadirá el sempiterno, referido al mundo, dejando el adjetivo «perpetuo» para significar la temporalidad humana:

«Quod ut melius intelligitur, quid proprie sit eternum, quid sempiternum quid perpetuum dicamus. Et est eternum quod caret principio et fine ut Creator; sempiternum quod, carens fine, habet principium non in tempore sed cum tempore ut mundus; perpetuum quod incipit in tempore et caret fine ut anima hominis» (Op. cit. XLIII, p. 111).

La diferencia entre sempiternum aplicado al mundo y perpetuum aplicado al alma humana está en las preposiciones in y cum: el sempiternum ha surgido cum tempore, mientras que las realidades perpetuas se han originado in tempore. 
Para Guillermo este mundo es bueno porque ha sido querido por Dios desde el principio. Así se descarta toda visión dualista o maniquea ya que el mal no ha sido producto de la creación, sino de la corrupción posterior de realidades que nunca llegarán a igualarse al Creador.

En Timeo 30a aparece el desorden o confusión primera que algunos asocian al caos primigenio de las cosmogonías arcaicas griegas. ¿Por qué estabam los elementos in prima creatione en tal estado de agitación?, se pregunta Guillermo. No le vale la respuesta piadosa: "ad caritatem et dilectionem Creatoris dilatandam» por las razones apuntadas anteriormente: ¿a quién iba a manifestar Dios su capacidad creadora?

La única respuesta posible es que los elementos fueron creados al principio con todas sus cualidades sustanciales como se dan ahora, pero sin las accidentales. Estas diferencias accidentales permiten explicarnos la confusión primera según el versículo del Génesis, I,2: «Terra erat inanis et vacua et tenebras erant super faciem abyssi».

A partir del movimiento producido por las cualidades de los elementos primarios, se fueron formando los seres corpóreos, entre ellos las estrellas, de carácter ígneo, que iniciaron un movimiento a partir del cual comenzó sa calentarse el aire y después el agua y fueron apareciendo las aves y los peces. Posteriormente aparecieron los animales terrestres.

$\mathrm{Al}$ abordar la creación del hombre, trata de interpretar Génesis II,7: «Formavit Deus hominem ex lino terrae et inspiravit in faciem eius spiraculum vitae». Aunque el cuerpo humano ha sido creado a partir del barro terrestre, el alma, por el hecho de ser levis y munda, no ha sido hecha de barro sino que el Creador se la ha conferido directamente al ser humano. Así el alma humana es para Conches una sustancia armoniosa superior a los demás seres:

«Unde cum diversa melancolica facta sint animalia et infinita flegmatica et colerica, unus solus homo formatus est quia ut ait Boetius in 'Arismetica': Omnis qualitas pauca est et finita, inequalitas numerosa et multiplex» (Glosae LII, p. 121-122).

Con estas ideas muy resumidas, tomadas de las Glosae de Guillermo al Timeo hemos querido presentar otra lectura chartriana del diálogo platónico, que, aunque con un enfoque diferente al de Teodorico, participa de su mismo espíritu optimista y claramente naturalista.

\section{CONCLUSIÓN}

Hemos visto cómo el camino seguido por estos dos autores dentro de este programa de concordia doctrinal es bastante diferente y hasta opuesto: mientras Teodorico parte del texto de Génesis y trata de hacer una exégesis racional iuxta physicam, Guillermo de Conches glosa el Timeo y trata de acomodarlo, dentro también de una línea naturalista, a la cosmovisión cristiana. El resultado final es la confirmación y consolidación de esta mentalidad chartreana, concordista, optimista, abierta a las nuevas doctrinas y a los conocimientos científicos del momento. 
Como conclusión del análisis de esta lectura destacaremos algunos temas de debate y reflexión que constituyen algunos de los púntos nodales de la síntesis chartreana.

1) El Demiurgo y el Creador. Resulta difícil asimilar el Demiurgo platónico al Creador ya que éste era impensable dentro de un contexto pagano que carecía de un concepto claro del origen ex nihilo.

Mientras la demiurgia platónica se inspira en el desarrollo de la técnica en Grecia, el Creador no actúa como artesano o artista por no existir ninguna realidad anterior a él. Por ello adquiere relevancia filosófica el tema de la eternidad de la materia.

Existe además otra diferencia acusada entre estos dos conceptos: mientras el Creador inaugura con su obra lo que en teología cristiana se denomina «la historia de la salvación», la narración del Timeo tiene un carácter mitológico carente de toda dimensión histórica.

A pesar de estas diferencias, los chartreanos intentaron aproximar ambas figuras, evitando destacar lo que las separaba.

2) La Trinidad en la Creación. Los filósofos de Chartres, movidos por el espíritu de la Carta a los Romanos: la búsqueda del Creador a través de las cosas visibles, pensaron que la creación fue una obra común de las tres personas de la Trinidad y trataron de descibrir la función de cada una de ellas. Se corría el peligro de considerar a la Trinidad de una forma un tanto extrovertida y diluir el misterio trinitario en una serie de tareas cosmogónicas.

Hay en todas estas formulaciones chartreanas un planteamiento que me atrevería a calificar, con algunos matices, de gnóstico. Mientras en Abelardo se acentúa más el carácter alegórico y teológico, en Guillermo se enfatiza más el carácter mundano del poder, la sabiduría y la bondad de Dios, sin ánimo de salirse de la ortodoxia sino como una ilustración racional del dogma trinitario, ilustración que en autores como Gillermo adquiere tonos claramente naturalistas.

3) La materia primordial o hyle. Aunque todos están de acuerdo en afirmar la creación de la materia frente a la concepción pagana de una materia eterna, existen diferentes formulaciones de la misma: desde la massa confussionis de ese caos primigenio con acentos ovidianos de Bernardo Silvestre hasta la materia como possibilitas frente a la forma de Teodorico pasando por una concepción más próxima al receptaculum platónico en Conches.

Con todas estas metáforas: silva, hyle, receptaculum, nodriza o madre, debe quedar claro que se trata de un concepto enormemente ambiguo y oscuro, debido a la falta de concreción del platonismo y del aristotelismo en relación con una problemática tan alejada del mundo transcendente de las Ideas.

4) El Alma del mundo. El puesto ocupado por el Alma del mundo en la tradición platónica es un puesto intermedio entre los seres superiores, inmutables e invisibles y los seres inferiores mudables y divisibles. Al participar de ambos mundos trataba de servir de elemento de equilibrio entre ellos con el fin de evitar un dualismo acusado.

De ahí que recogiera matices de otras tradiciones, desde el principio vital del animismo arcaico griego al «lógos-pūr» estoico, principio racional de todo devenir, o el spiritus que todo lo llena recogido por Virgilio en la Eneida (L. VI). 
El pensamiento cristiano consideró siempre este concepto como una muestra de la presencia especial de Dios en el mundo, partiendo de referencias bíblicas como Génesis I,2: «et Spiritus Dei ferebatur super aquas» hasta Hechos 17,28: «In ipso enim vivimus et movemur et sumus...». Por eso no es de extrañar que, dentro de una línea de concordia doctrinal, existiera una tendencia a la identificación con el Espíritu Santo. Pero como tal identificación se apartaba de la ortodoxia, optaron, bajo el influjo árabe, por considerar al Alma del mundo como la expresión más depurada de la idea de Naturaleza.

Para un pensador cristiano con ánimo concordista como es el pensador chartreano, esta naturalización del Alma del Mundo es contemplada como una prolongación en el tiempo de la actividad creadora de Dios, de acuerdo con el versículo de Juan VI,63:

«Spiritus est qui vivificat» o la frase del Apóstol: «Deus est, enim, qui operatur in vobis velle et perficere» (Filip. II,13).

5) El concepto de Naturaleza . Este proceso de naturalización del concepto de Alma del Mundo, que tiene lugar a partir del Concilio de Sens (1140) que condenó la tesis de la identificación con el Espíritu Santo, va a desembocar en una concepción de la Natura como principio del devenir físico, constituido por el conjunto de las causas segundas que han recibido de Dios la suficiente autonomía para desarrollar y completar su obra creadora.

Es la idea de un naturalis vigor que en Teodorico todavía queda separada del Alma del mundo, pero que en Guillermo representa un principio de toda clase de vida, auténtica fuerza generativa:

«Natura est ignis artifex ex quadam vi ut procedens in res sensibiles procreandas; Physici namque dicunt omnia ex calore et humore procreari» (Conches. Didascalion I,11, PL 176, col. 747).

Esta personificación de la Natura artifex como mater generationis había contado con precedentes clásicos muy claros desde Cicerón y Plinio el joven hasta Macrobio. Aparece también en Virgilio y en el Asclepius.

Finalmente, Bernardo Silvestre, el poeta cosmológico por excelencia de la escuela de Chartres, acenturará esta concepción autónoma de la Natura, y con su poesía contribuirá a personificarla, describiéndola con rasgos de diosa pagana de la fecundidad, cooperadora del Opus creatoris, que manifiesta exteriormente las riquezas encerradas en la Mente divina.

\section{BIBLIOGRAFIA}

\section{Fuentes:}

CALCIDIO, Timeus a Calcidio translatus Commentarioque instructus. Ed. J. H. Waszink (Plato Latinus IV. London-Leiden, 1975. 
GUILLERMO DE CONCHES, Glosae super Platonem. Ed. E. Jeauneau, Paris, 1965.

TEODORICO DE CHARTRES, De sex dierum operibus. Ed. N. Häring, AHDLMA 22 , 1956, pp. 137 216.

\section{Literatura secundaria:}

FLATTEN, H., «Die 'materia primordialis' in der Schule von Chartres» en Archiv für Gesichte der Philosophie, 1931, pp. 58-65.

GREGORY, T., Anima mundi. La filosofia di Guglielmo di Conches e la scuola di Chartres. Firenze, 1955. Platonismo medievale. Roma, 1958.

JEAUNEAU, E., «L'usage de la notion d'integumentum à travers les gloses de Guillaume de Conches», AHDLMA 24, 1958, pp. 35-100. «Note sur l'Ecole de Chatres» en Studi Medievali, 1964, pp. 82165. «Simples notes sur la cosmogonie de Thiérry de Chartres» en Sophia 23, 1955, pp. 172-183.

MACCAGNOLO, C., Rerum universitas. Saggio sulla filosofia di Teodorico di Chatres. Firenze, 1976. Il Divino e il Megacosmo. Testi filosofici et scientifici della Scuola di Chatres. Rusconi. Milano, 1980.

PARENT, J., La doctrina de la création dans l'Ecole de Chartres. Etude et textes. Publications de l'Institut d'Etudes Médiévales d'Ottawa. Paris-Ottawa, 1938. 